\title{
$1 \quad$ Modelling brain representations of abstract concepts
}

2

3 Daniel Kaiser ${ }^{1, *}$, Arthur M. Jacobs ${ }^{2,3}$, Radoslaw M. Cichy ${ }^{2,4,5}$

4

$5 \quad{ }^{1}$ Department of Psychology, University of York

$6 \quad{ }^{2}$ Department of Education and Psychology, Freie Universität Berlin

$7 \quad{ }^{3}$ Center for Cognitive Neuroscience Berlin, Freie Universität Berlin

$8 \quad{ }^{4}$ Berlin School of Mind and Brain, Humboldt-Universität zu Berlin

$9 \quad{ }^{5}$ Bernstein Center for Computational Neuroscience Berlin

10

\section{1 *Correspondence:}

12 danielkaiser.net@gmail.com 


\section{Abstract}

16 Abstract conceptual representations are critical for human cognition. Despite their 17 importance, key properties of these representations remain poorly understood. Here, 18 we used computational models of distributional semantics to predict multivariate fMRI 19 activity patterns during the activation and contextualization of abstract concepts. We

20 devised a task in which participants had to embed abstract nouns into a story that 21 they developed around a given background context. We found that representations 22 in inferior parietal cortex were predicted by concept similarities emerging in models 23 of distributional semantics. By constructing different model families, we reveal the 24 models' learning trajectories and delineate how abstract and concrete training 25 materials contribute to the formation of brain-like representations. These results 26 inform theories about the format and emergence of abstract conceptual 27 representations in the human brain. 


\section{Introduction}

31 The use of conceptual knowledge is one of the foundations of human intelligence. On

32 the neural level, concepts are represented in a complex network of brain regions

33 (Binder et al., 2009). Fueled by novel computational models of distributional 34 semantics, researchers have recently started to unravel the format of concept representations in this neural network. By harnessing linguistic co-occurrence statistics, these models not only capture representations of concepts from written and spoken language (Deniz et al., 2019; Huth et al., 2016; Just et al., 2010; Mitchell et al., 2008), but also predict representations of novel concepts (Pereira et al., 2019).

However, these recent advances in understanding the representations of conceptual knowledge largely hinge on the study of concrete concepts. Only few studies have investigated how abstract concept representations are organized (Anderson et al., 2014; Vargas \& Just, 2020; Wang et al., 2018). To date, key questions about the emergence and the format of these representations remain heavily debated (Borghi et al., 2017).

Here, we model brain representations that support the activation and contextualization of abstract concepts. We recorded fMRI while participants were tasked with embedding abstract words into a background context. By relating brain activations during this task to targeted models of distributional semantics, we shine a new light on the format of abstract concept representations in the human brain. 


\section{Results and Discussion}

In an fMRI experiment, we visually presented 61 abstract German nouns (see Materials and Methods). Participants $(n=19)$ read these words and silently embedded them into a coherent story that they were developing around a prespecified contextual background (Figure 1a).

Cortical responses were modelled in a representational similarity searchlight analysis (Figure 1b), in which we extracted similarity relations among the words across the whole cortex (see Materials and Methods). We modelled these similarities using a word2vec model of distributional semantics (Mikolov et al., 2013), trained on a 45million sentence corpus (SdeWaC; Faaß \& Eckhart, 2013).

This model predicted brain activations in the left inferior parietal cortex (IPC; Figure 1c), most prominently covering the angular gyrus, but extending into the superior parietal and middle occipital cortices (158 voxels, peak: -36/-58/46, t[18]=6.16), and in right IPC, with an anterior cluster primarily in the supramarginal gyrus (91 voxels, peak: 45/-46/58, t[18]=4.91), and a posterior cluster in the angular gyrus (35 voxels, peak: $36 /-73 / 40, t[18]=4.80)$. These results show that bilateral IPC represents semantic similarities of abstract concepts. They further suggest IPC as a cortical hub for the activation and contextualization of abstract concepts.

a

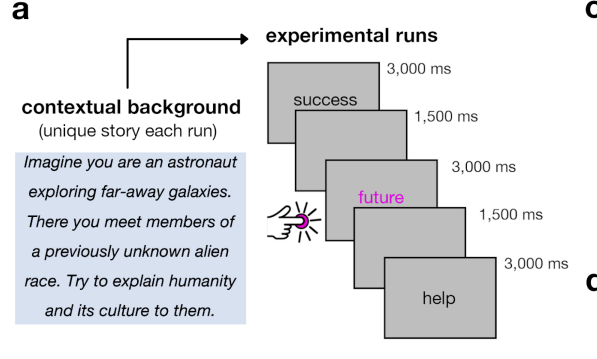

b moving searchlight sphere from word2vec model

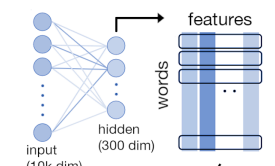

$\rightarrow$

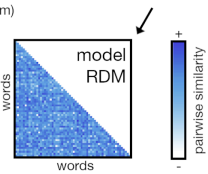

C

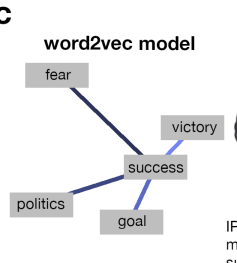

d

control: affect grid
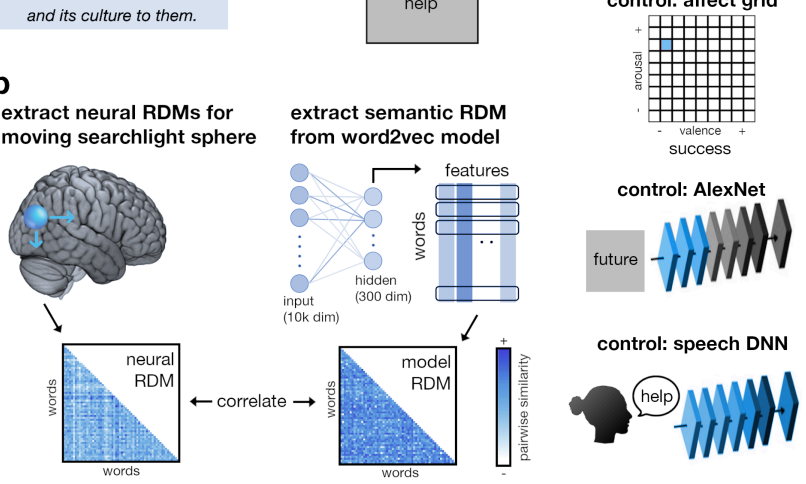
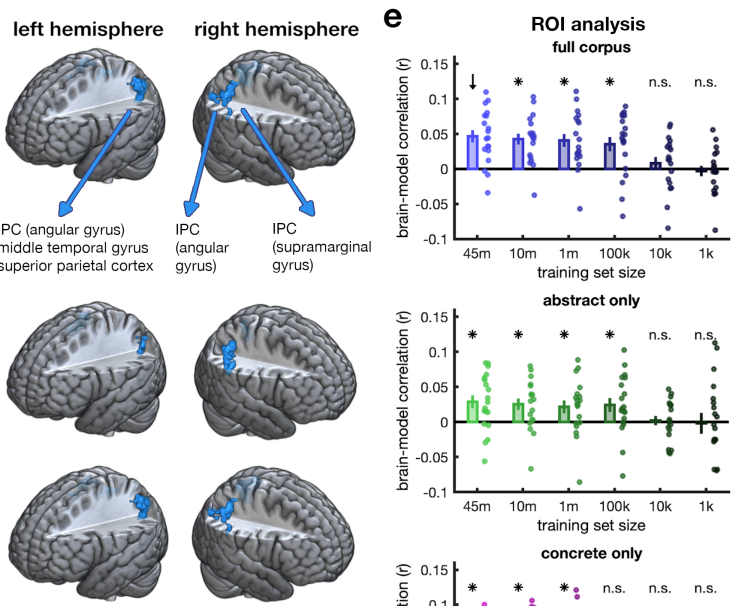

concrete only

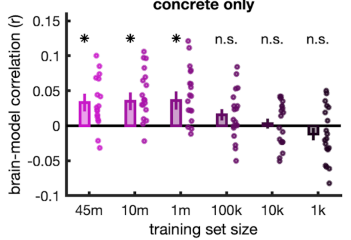


Figure 1. Representation of abstract concepts in parietal cortex. a) Participants completed 10 runs of $\mathrm{fMRl}$ recordings. Before each run, they established a unique background context in their mind and were then asked to silently narrate a story of their own making that included the subsequently presented 61 abstract words, in random order (all German word stimuli in Supplementary Information). b) In a searchlight analysis, representational dissimilarity matrices (RDMs) were extracted (i) from the brain data, by pairwise correlations among localized activity patterns, and (ii) from a word2vec model of distributional semantics, by pairwise correlations among hidden-layer activations. c) Correlating the neural and model RDMs revealed clusters in bilateral inferior parietal cortex (IPC), primarily covering the angular gyrus. Brain maps are thresholded at $p_{\text {voxe }}<0.001$ (uncorrected) and $p_{\text {cluster }}<0.05$ (FWE-corrected). d) These clusters persisted when repeating the analyses while partialing out the effects of emotional word content (using affect grids), visual wordform (using a visualcategorization DNN), and auditory properties of the spoken words (using a speechrecognition $D N N)$. e) Within the IPC cluster defined on the full 45-million sentence model (marked by an arrow), we compared model families trained on different corpus sizes and on only abstract or concrete words, respectively. Brain-like representations emerged in models that were trained on as little as 100,000 sentences and on either abstract or concrete embeddings. Dots show individual-participant data, error bars denote SEM, asterisks represent $p<0.05$ (FDR-corrected).

\section{Controlling for emotional and sensory word properties}

Some researchers have argued that abstract concepts are represented through their grounding in the emotional domain (Kousta et al., 2011; Vigliocco et al., 2011). To test whether IPC representations are indeed driven by the words' emotional content, we re-performed the analysis while partialing out valence and arousal ratings (see Materials and Methods). We still found clusters in left (36 voxels, peak: -33/-64/31, $\mathrm{t}[18]=4.73$ ), and right (108 voxels, peak: 63/-40/31, t[18]=7.30) IPC, suggesting that the emotional content is insufficient to explain abstract concept representation in parietal cortex. 
107 IPC is also sensitive to sensory properties, such as visual form (Freud et al., 2018) and

108 phonological speech attributes (Hartwigsen et al., 2010). However, when repeating

109 the analysis while partialing out early activations in a visual-categorization deep neural

110 network (DNN; see Materials and Methods), we still found clusters in the left (157 111 voxels, peak: $-36 /-58 / 46, t[18]=6.07$ ) and right (anterior: 76 voxels, peak: 51/-49/52, $112 \mathrm{t}[18]=4.70$; posterior: 32 voxels, peak: 39/-76/40, $t[18]=4.82)$ IPC. Similarly, when

113 controlling for activations obtained from a speech-recognition DNN (see Materials and 114 Methods), we still found clusters in left (113 voxels, peak: $-33 /-64 / 31, t[18]=5.43$, $115 \mathrm{p}_{\text {cluster }}<0.001$ ), and right (53 voxels, peak: 48/-46/55, $t[18]=4.68$ ) IPC. These results 116 show that sensory properties are unrelated to IPC representations of abstract 117 concepts.

Trajectories towards brain-like representations

121 Our observation that the word2vec model and IPC share abstract concept representations led us to ask how the model acquires this property. To test whether co-occurrence statistics are acquired incrementally over increasing experience with human language, we devised a word2vec model family whose members were trained on staggered amounts of data, from the full 45-million sentence corpus down to fragments as small as 1,000 sentences. We then evaluated how well models trained on less data could still predict representations in the IPC cluster that yielded the best correspondence with the full 45-million sentence model (see Materials and Methods).

This analysis revealed decreasing correspondence with decreasing training data

131 (mean $r=0.74, t[18]=5.89, p<0.001$ ). Nonetheless, a model trained on only 100,000 132 sentences ( $0.2 \%$ of the corpus) still predicted IPC representations well $(\mathrm{t}[18]=3.46$, $133 \mathrm{p}_{\mathrm{FDR}}=0.002$; comparison to full model: $\left.\mathrm{t}[18]=2.15, \mathrm{p}=0.045\right)$, whereas models trained 134 on smaller training sets did not (Figure 1d). These results show that brain-like 135 representations are learned through linguistic co-occurrence statistics, which can 136 emerge already from a (relatively) modest degree of training experience. 
139 Some theorists argue that the meaning of abstract concepts needs to be derived

140 through the activation of related concrete concepts, which are in turn grounded in 141 sensory experiences (Kiefer \& Pulvermüller, 2012; Lakoff, 2008). This view prompts

142 the hypothesis that representations of abstract concepts originate primarily from co143 occurrence statistics between abstract and concrete words, rather than among 144 abstract words alone. To test this hypothesis, we trained word2vec models on 145 subsets of the 45-million sentence corpus that we devised to consist of abstract or 146 concrete words only (see Materials and Methods).

148 Models trained on abstract-only and concrete-only corpora both predicted 149 representations in IPC (Figure 1e). Reproducing the previous pattern of results, we 150 found that models trained on larger fractions of the corpus predicted representations 151 better (abstract only: mean $r=0.36, t[18]=2.55, p=0.010$; concrete-only: mean $r=0.73$, $152 \mathrm{t}[18]=4.32, \mathrm{p}<0.001)$. Interestingly, representations were modelled equally well by the 153 most extensively trained abstract-only $\left(\mathrm{t}[18]=3.09, \mathrm{p}_{\mathrm{FDR}}=0.010\right)$ and concrete-only 154 models $\left(t[18]=2.60, p_{F D R}=0.018\right.$; comparison: $\left.t[18]=0.50, p=0.62\right)$, suggesting that 155 brain-like representations of abstract concepts can emerge from either abstract or 156 concrete semantic embeddings.

\section{Conclusions}

160 Our findings yield multiple key insights into abstract concept representation:

162 First, our findings provide novel evidence for IPC as a core area for concept coding 163 (Binder et al., 2009). Using a task that required participants to activate and 164 contextualize abstract concepts, we identify the angular gyrus as a critical hub for the 165 dynamic use of abstract knowledge, consistent with the view that this region plays a 166 key role in combinatory linguistic processing (Graessner et al., 2021; Price et al., 2015; 167 Pylkkänen, 2019). Such combinatory processing is critical in real-life situations that 168 require applying abstract knowledge in a situational and meaningful way.

170 Second, our study informs theories of abstract knowledge representation (Borghi et 171 al., 2017). We show that the meaning of abstract concepts can be derived from 
172 distributional word embeddings in natural language (Wang et al., 2018). Interestingly,

173 the organization of abstract concepts, as found in our brains, can be derived from

174 linguistic embeddings in both abstract and concrete realms of knowledge. This result

175 shows that despite their representational dissimilarities (Binder et al., 2005; Wang et

176 al., 2010), abstract and concrete concepts may derive their meaning through shared

177 principles. By contrast, our data do not provide evidence for theories suggesting that

178 abstract concepts are coded solely through emotional associations (Vigliocco et al.,

1792011 ) or the activation of related concrete concepts (Harpaintner et al., 2020; Lakoff,

180 2008). They rather suggest that abstract knowledge is represented through concept

181 relationships in the language processing system.

183 Third, our results establish that computational models of distributional semantics

184 acquire brain-like abstract concept representations by harnessing co-occurrence

185 statistics from linguistic experience. Although massive corpora are immensely popular

186 for modelling language organization, our analyses of model learning trajectories show

187 that brain-like representations can emerge from much smaller training sets of only

188100,000 sentences. Future work could map out the emergence of more fine-grained

189 information along these model learning trajectories to predict human concept learning

190 and development (Vigliocco et al., 2018).

192 Finally, our study highlights that computational models - through systematic 193 manipulation of model training regimes - can yield targeted insights into the 194 emergence and format of concept representations. Moving ahead, future studies 195 could not only refine training regimes but also comprehensively manipulate 196 fundamental model parameters such as architecture, learning material and learning 197 procedure (Cichy \& Kaiser, 2019). Such model-based analyses may yield further fine198 grained insights into how our brain represents abstract knowledge. 


\section{Materials and Methods}

\section{Participants}

204 Nineteen healthy adults (mean age 28.8 years, SD=6.1; 10 female) with normal or corrected-to-normal vision completed the experiment. All of them were right-handed and native German speakers. Participants provided informed consent and received monetary reimbursement or course credits for participation. Participants were recruited from the online participant database of the Berlin School of Mind and Brain (Greiner, 2015). All procedures were approved by the local ethical committee and were in accordance with the Declaration of Helsinki.

\section{Stimuli and Paradigm}

The stimulus set consisted of 61 abstract German nouns. All words and their English translations can be found in the Supplementary Information (Table S1).

During the fMRI experiment, participants completed 10 runs. Before each run, participants read through one of 10 contextual background stories. All texts and their

219 English translations can be found in the Supplementary Information (Table S2). Participants were asked to mentally image themselves being in the scenario outlined in the text. After reading through the text, participants were instructed to use the subsequently presented words in the upcoming run to mentally narrate a story that incorporates the words as they are shown on the screen. They were instructed that it

224 is completely up to them how the story unfolds as long as they use all the words in 225 their story. Stories were chosen to be emotionally engaging to increase participants' immersion into the task. The order of the 10 stories was randomized for every participant.

229 Each run contained 61 experimental trials. On each trial, one of the abstract words was shown for 3 seconds, in black Arial font on a gray background. Trials were

231 separated by an inter-trial interval of 1.5 seconds, during which a fixation cross was 232 shown. In addition to the experimental trials, each run included 14 fixation trials, where 
only the fixation cross was shown throughout the trial. Trial order was randomized

234 within each run.

To ensure that participants paid attention to the words, we introduced a simple manual task: In each run, 7 of the word were shown in pink color and participants had to press a button whenever they saw one them.

Runs started and ended with brief fixation periods; each run lasted 5:48 minutes. The stimulation was back-projected onto a translucent screen at the end of the MRI scanner bore and controlled using the Psychtoolbox (Brainard, 1997).

Additionally, prior to the experiment, each participant completed a practice run (using a background text different from the ones used in the experiment).

Two participants completed a version of the experiment that differed in two aspects: the inter-trial interval was $1 \mathrm{~s}$ instead of $1.5 \mathrm{~s}$ and no behavioral task was included.

\section{MRI acquisition and preprocessing}

MRI data was acquired using a 3T Siemens Tim Trio Scanner equipped with a 12channel head coil. T2*-weighted gradient-echo echo-planar images were collected as functional volumes (TR=2s, $T E=30 \mathrm{~ms}, 70^{\circ}$ flip angle, $3 \mathrm{~mm}^{3}$ voxel size, 37 slices, $20 \%$ gap, 192mm FOV, 64×64 matrix size, interleaved acquisition). Additionally, a T1weighted image (MPRAGE; $1 \mathrm{~mm}^{3}$ voxel size) was obtained as a high-resolution anatomical reference. Preprocessing was done in MATLAB using SPM12

258 (www.fil.ion.ucl.ac.uk/spm/). The functional volumes were realigned and coregistered

259 to the T1 image. The T1 image was normalized to MNI-305 standard space to obtain 260 transformation parameters used to normalize participant-specific results maps (see 261 below). 
To quantify neural representations, we used multivariate representational similarity analysis (RSA) (Kriegeskorte et al., 2008). In RSA, neural representations are first characterized by means of their pairwise similarity structure (i.e., how similarly each stimulus is represented with each other stimulus). The pairwise dissimilarities between neural representations are organized in neural representational dissimilarity matrices (RDMs) indexed in rows and columns by the experimental conditions compared. Then, the neural similarity structure (i.e., the neural RDMs) are correlated to model RDMs, which capture different aspects of the conditions' similarity. Significant correlations between the neural RDMs and these model RDMs indicate that the aspect of similarity conveyed by the model is represented in the brain.

\section{Extracting neural dissimilarity}

Separately for each participant and each run, we first modeled the functional MRI data in a general linear model (GLM) with 67 predictors (61 predictors for the 61 words, and 6 predictors for the 6 movement regressors obtained during realignment). From these GLMs, we obtained 610 beta weights of interest for every voxel, which quantified the voxel's activation to each of the 61 words in each of the 10 runs. All further analyses were carried out using a searchlight approach (Kriegeskorte et al., 2006), that is, analyses were done repeatedly for a spherical neighborhood (3-voxel radius) centered on each voxel across the brain. This approach allowed us to quantify and model neural representations in a continuous and unconstrained way across brain space.

For each searchlight neighborhood, neural RDMs were created based on the similarity of multi-voxel response patterns, using the CoSMoMVPA toolbox (Oosterhof et al., 2016). Within each neighborhood, we extracted the response pattern across voxels evoked by each word in each run. We then performed a cross-validated correlation analysis (Haxby et al., 2001). For this analysis, the data were repeatedly split into two

294 halves (all possible 50/50 splits; results were later averaged across these splits) and 295 the response patterns for each word were averaged within each half. For each pair of 296 words, we then computed two correlations: (i) within-condition correlations were 
one half of the data with the response patterns evoked by the same word in the other half of the data, and (ii) between-condition correlations were computed by correlating the response patterns evoked by each of the two words in one half of the data with the response patterns evoked by the other word in the other half of the data. By subtracting the between-correlations from the within-correlations for each pair of words, we obtained an index of how dissimilar two words are based on the response patterns they evoked in the current searchlight neighborhood. Repeating this analysis for each pair of words yielded a $61 \times 61$ neural RDM for each searchlight.

\section{Modelling neural dissimilarity}

To model the semantic representation of the abstract words, we used a word2vec computational model of distributional semantics (Mikolov et al., 2013). The model was

311 trained on the SdeWaC corpus, which contains 45 million German sentences (Faaß \& 312 Eckhart, 2013) using the gensim library (https://github.com/RaRe-

313 Technologies/gensim). The model hyperparameters were the following: dimensions = 314300 , model type $=$ skipgram, windowsize $=5$, minimum count $=1$, iterations $=50$. For 315 each word in the corpus, this model yields a vector representation that indicates its 316 position in a 300-dimensional vector space. Distances in this vector space reflect similarities in contextual embeddings. We then created a $61 \times 61 \mathrm{RDM}$ based on the pairwise correlations of the 300 vector-space features for each of the words used in the experiment.

To establish correspondences between the model and the brain data, the model RDMs were correlated with the neural RDMs for each searchlight. These correlations were then Fisher-transformed and mapped back to the searchlight center. We thereby obtained brain maps of correspondence between each model and the neural data. For each participant, these maps were warped into standard space by using the normalization parameters obtained during preprocessing. 
As an emotional content model, we used participants' responses in an affect grid task, where 20 participants (partly including the participants in the current experiment) concurrently rated each word's valence and arousal by selecting one compartment of a $9 \times 9$ grid (Russell et al., 1989). From these data, we created two RDMs: (i) a valence RDM, whose entries reflected pairwise absolute difference in the words' valence ratings and (ii) an arousal RDM, whose entries reflected pairwise absolute difference in the words' arousal ratings. The valence and arousal RDMs were mildly correlated with each other $(r=0.19)$ and with the different word2vec model RDMs (all $r<0.24)$. The words' similarity in valence an arousal did not significantly predict brain activations in a searchlight analysis.

As a visual word form model, we used activations in the three earliest convolutional layers an AlexNet DNN pre-trained on object recognition (Krizhevsky et al., 2012; Vedaldi \& Lenc, 2015), which have been shown to capture representations of simple visual attributes in visual cortex (Cichy et al., 2016). We printed the 61 words as they appeared in the experiment on a $225 \times 225$ pixel gray image background and fed the resulting images to the background. The resulting network activations were used to construct model RDMs. For each of the first three convolutional layers of the network, the RDM was constructed by computing pairwise distances (1-correlation) between layer-specific activation vectors. The visual DNN RDMs were only very weakly correlated with the word2vec model RDMs (all $r<0.1$ ). Searchlight analyses revealed that the first three layers of the visual DNN predicted activations in bilateral posterior visual cortex, including fusiform cortex (see Supplementary Information, Figure S1).

As a model of auditory, phonetic word similarity, we used activations in a DNN model of auditory speech recognition (Kell et al., 2018). We obtained spoken versions of the 61 words from the ttsmp3 webpage (https://ttsmp3.com/text-to-speech/German/). The sound files were resized to a length of 2 seconds by right-padding them with zeros, transformed into a cochleagram representation, and then passed through the speech recognition branch of the DNN. The resulting network activations were used to construct model RDMs. For each of the seven layers of the network, the RDM was constructed by computing pairwise distances (1-correlation) between layer-specific activation vectors. The auditory DNN RDMs were only weakly correlated with the 
363

364

365

366

367

368

369

370

371

372

373

374

375

376

377

378

379

380

381

382

383

384

385

386

387

word2vec model RDMs (all $r<0.16$ ). Searchlight analyses revealed that the early layers of the auditory DNN, because of the correlation between word length and speech duration, also predicted activations in bilateral posterior visual cortex. By contrast, the last layer of the network specifically predicted activations in left middle temporal gyrus (see Supplementary Information, Figure S1).

To control for emotional and sensory properties, we performed searchlight analyses relating the neural RDMs and the word2vec model RDMs as before, while we partialed out the two emotion predictor RDMs, the three visual DNN predictor RDMs, or the seven auditory DNN predictor RDMs, respectively. All other aspects of the analyses remained identical.

\section{$\underline{\text { Region of interest analyses }}$}

For further dissecting the representations in left parietal cortex, we specifically focused on this area in a region-of-interest $(\mathrm{ROI})$ analysis. The IPC clusters that showed significant correspondence with the word2vec model in the main analysis were chosen as the ROI. Neural RDMs were generated from pairwise correlations of activity patterns across all voxels in the ROI; the procedure was otherwise identical to the procedure applied in the searchlight analysis (see above).

As ROI definition was done on the basis of the model that was trained on the full 45million sentences SDeWaC corpus, we never evaluated this model statistically in our $\mathrm{ROI}$ analysis. We instead probed the correspondence between neural RDMs in the $\mathrm{ROI}$ and RDMs built from a set of different word2vec model families whose training regimes differed in important aspects.

To probe the behavior of our word2vec model with changes in training set, we created a model family whose members were trained on different amounts of data. Models were trained on different fragments of the corpus (containing $45 \mathrm{~m}, 10 \mathrm{~m}, 1 \mathrm{~m}, 100 \mathrm{k}$, $10 \mathrm{k}$, or $1 \mathrm{k}$ sentences). Each of these fragments corresponded to the first $n$ sentences in the corpus (e.g., the $1 \mathrm{k}$ model comprised the first 1,000 sentences). We thereby 
ensured that the smaller fragments were always completely included in the larger ones.

Additionally, we constructed a model family whose members were trained on abstract words and a model family whose members were trained on concrete words. Members in each family differed by the amount of data they were trained on (as outlined above). Abstract and concrete words were defined on the basis of the abstractnessconcreteness scale of the IMS norms (https://www.ims.unistuttgart.de/en/research/resources/experiment-data/affective-norms) (Köper \& im Walde, 2017). For the abstract-only models, we chose words that had a $z$-value of $<0$ on the on this scale and removed all other words from the corpus; this left us with $\sim 65$ million words ( $4 \%$ of the corpus). For the concrete-only models, we chose words that had a z-value of $>0$ and removed all other words from the corpus; this left us with $\sim 280$ million words ( $18 \%$ of the corpus). Note that for the concrete-only model, the 61 abstract words were also left in the corpus, so that relationships between them and the concrete words could be obtained. For all models of each model family, we extracted a $61 \times 61$ RDM, which was then correlated with the neural RDM extracted for

\section{Statistical testing}

For the searchlight analyses, to detect spatial clusters in which the neural data were explained by the different representational models, we performed one-sided t-tests against zero across participants, separately for each voxel in the correlation maps. The resulting statistical maps were thresholded at the voxel level at $p_{\text {voxel }}<0.001$ (uncorrected) and at the cluster level at $p_{\text {cluster }}<0.05$ (family-wise error corrected, as implemented in SPM12).

For the cross-validated ROI analyses, ROls were repeatedly defined in 18 participants, using the same statistical thresholding as in the full searchlight analysis

425 ( $p_{\text {voxel }}<0.001, \quad p_{\text {cluster }}<0.05$ FWE-corrected). Correlations between neural RDMs 426 extracted from the ROls and model RDMs were then evaluated using one-sided t- 
tests against zero across participants. Results were corrected for multiple comparisons across the different training corpus sizes using FDR corrections.

\section{Data availability}

Data are publicly available on OSF (https://doi.org/10.17605/OSF.IO/FTBJQ). For other materials, please contact the corresponding author.

\section{Acknowledgements}

Thanks to Raphael Leuner and Kshitij Dwivedi for help with the computational analyses. D.K. and R.M.C. are supported by DFG grants (KA4683/2-1, Cl241/1-1, Cl241/3-1, Cl241/7-1). R.M.C. is supported by an ERC Starting Grant (ERC-2018-StG 803370).

\section{References}

Anderson AJ, Murphy B, Poesio M. (2014). Discriminating taxonomic categories and domains in mental simulations of concepts of varying concreteness. J Cogn Neurosci, 26, 658-681.

Binder JR, Westbury CF, McKiernan KA, Possing ET, Medler DA. (2005). Distinct brain systems for processing concrete and abstract concepts. J Cogn Neurosci, 17, 905-917.

Binder JR, Desai RH, Graves WW, Conant LL. (2009). Where is the semantic system? A critical review and meta-analysis of 120 functional neuroimaging studies. Cereb Cortex, 19, 2767-2797.

Borghi AM, et al. (2017). The challenge of abstract concepts. Psychol Bull, 143, 263292.

Brainard DH. (1997). The psychophysics toolbox. Spat Vis, 10, 433-436. 
Cichy RM, Khosla A, Pantazis D, Torralba A, Oliva A. (2016). Comparison of deep neural networks to spatio-temporal cortical dynamics of human visual object recognition reveals hierarchical correspondence. Sci Rep, 6, 27755.

Deniz F, Nunez-Elizalde AO, Huth AG, Gallant JL. (2019). The representation of semantic information across human cerebral cortex during listening versus reading is invariant to stimulus modality. J Neurosci, 39, 7722-7736.

Faaß G, Eckhart K. (2013). SdeWaC - a corpus of parsable sentences from the web. In: Gurevych I, Biemann C, Zesch T (eds). Language processing and knowledge in the web. Lecture notes in computer science, vol. 8105. Springer, Berlin, Heidelberg.

Freud E, Culham JC, Plaut DC, Behrmann M. (2018). The large-scale organization of shape processing in the ventral and dorsal pathways. eLife, 6, e27576.

Graessner A, Zaccarella E, Hartwigsen G. (2021). Differential contributions of lefthemispheric language regions to basic semantic composition. Brain Struct Funct, 226, 501-518.

Greiner B. (2015). Subject pool recruitment procedures: organizing experiments with ORSEE. JESA, 1, 114-125.

Hartwigsen G, et al. (2010). Phonological decisions require both the left and right supramarginal gyri. Proc Natl Acad Sci USA, 107, 16494-16499.

Harpaintner M, Sim E-J, Trumpp NM, Ulrich M, Kiefer M. (2020). The grounding of abstract concepts in the motor and visual system: an fMRI study. Cortex, $124,1-22$.

Haxby JV, et al. (2001). Distributed and overlapping representations of faces and objects in ventral temporal cortex. Science, 293, 2425-2430.

Huth AG, de Heer WA, Griffiths TL, Theunissen FE, Gallant JL. (2016). Human natural speech reveals the semantic maps that tile human cerebral cortex. Nature, 532, 453-458.

Just MA, Cherkassky VL, Aryal S, Mitchell TM. (2010). A neurosemantic theory of concrete noun representation based on the underlying brain codes. PLoS One, 5, e8622.

Kell AJ, Yamins DL, Shook EN, Norman-Haignere SV, McDermott JH. (2018). A task-optimized neural network replicates human auditory behavior, predicts 
brain responses, and reveals a cortical processing hierarchy. Neuron, 98, 630-644.

Kiefer M, Pulvermüller F. (2012). Conceptual representations in mind and brain: Theoretical developments, current evidence and future directions. Cortex, 48, 805-825.

Köper M, im Walde SS. (2017). Improving verb metaphor detection by propagating abstractness to words, phrases and individual senses. Proceedings of the 1st workshop on sense, concept and entity representations and their applications, 24-30.

Kousta ST, Vigliocco G, Vinson DP, Andrews M, Del Campo E. (2011). The representation of abstract words: why emotion matters. J Exp Psychol Gen, 140, 14.

Kriegeskorte N, Goebel R, Bandettini P. (2006). Information-based functional brain mapping. Proc Natl Acad Sci USA, 103, 3863-3868.

Kriegeskorte N, Mur M, Bandettini P. (2008). Representational similarity analysis connecting the branches of systems neuroscience. Front Syst Neurosci, 2, 4.

Krizhevsky A, Sutskever I, Hinton GE. (2012). ImageNet classification with deep convolutional neural networks. Advances in Neural Information Processing Systems, 1097-1105.

Lakoff G. (2008). The neural theory of metaphor. In: Gibbs Jr, WR (ed). The Cambridge handbook of metaphor and thought. Cambridge University Press, Cambridge.

Mikolov T, Chen K, Corrado G, Dean J. (2013). Efficient estimation of word representations in vector space. arXiv, arXiv:1301.3781.

Mitchell TM, et al. (2008). Predicting human brain activity associated with the meanings of nouns. Science, 320, 1191-1195.

Oosterhof NN, Connolly AC, Haxby JV. (2016). CoSMoMVPA: Multi-modal multivariate pattern analysis of neuroimaging data in Matlab/GNU Octave. Front Neuroinform, 10, 20.

Pereira F, et al. (2019). Toward a universal decoder of linguistic meaning from brain activation. Nat Commun, 9, 1-13. 
521 Price AR, Bonner MF, Peelle JE, Grossman M. (2015). Converging evidence for the neuroanatomic basis of combinatorial semantics in the angular gyrus. $J$ Neurosci, 35, 3276-3284.

524 Pylkkänen L. (2019). The neural basis of combinatory syntax and semantics. Science, 355, 62-66.

Russell JA, Weiss A, Mendelsohn GA. (1989). Affect grid: a single-item scale of pleasure and arousal. J Personal Soc Psychol, 57, 493.

Vargas R, Just MA. (2020). Neural representations of abstract concepts: identifying underlying neurosemantic dimensions. Cereb Cortex, 30, 2157-2166.

Vedaldi A, Lenc K. (2015). MatConvNet - convolutional neural networks for Matlab. ACM International Conference on Multimedia.

Vigliocco G, et al. (2011). The neural representation of abstract words: the role of emotion. Cereb Cortex, 24, 1767-1777.

534 Vigliocco G, Ponari M, Norbury C. (2018). Learning and processing abstract words and concepts: insights from typical and atypical development. Top Cogn Sci, 10, 533-549.

Wang J, Conder JA, Blitzer DN, Shinkareva SV. (2010). Neural representation of abstract and concrete concepts: a meta-analysis of neuroimaging studies. Hum Brain Mapp, 31, 1459-1468.

540 Wang X, et al. (2018). Organizational principles of abstract words in the human brain. Cereb Cortex, 28, 4305-4318. 